\title{
Outdoor Face Recognition Using Enhanced Near Infrared Imaging
}

\author{
Dong Yi, Rong Liu, RuFeng Chu, Rui Wang, Dong Liu, and Stan Z. Li \\ Center for Biometrics and Security Research \& \\ National Laboratory of Pattern Recognition, \\ Institute of Automation, Chinese Academy of Sciences, Beijing 100080, China \\ http://www.cbsr.ia.ac.cn
}

\begin{abstract}
In this paper, we present a robust and accurate system for outdoor (as well as indoor) face recognition, based on a recently developed enhanced near-infrared (ENIR) imaging device. Using a narrow band NIR laser generator instead of LED lights for active frontal illumination, the ENIR device can provide face images of good quality even under sunlight. Experiments show that the ENIR system performs similarly to the existing NIR system when used indoors, but outperforms it significantly outdoors especially under sunlight.
\end{abstract}

Key words: Face recognition, near-infrared (NIR), imaging device, illumination, outdoor, statistical learning.

\section{Introduction}

Over the past few years, face recognition has more and more applications in our daily life such as access control, surveillance, information safety, humanmachine interaction and multimedia communication. However, for practical face recognition systems, the effect of variation in the lighting conditions is one of the most challenging problems [1]. As report in FRVT2002, the variation of outdoor lighting has a drastic affect on performance of face recognition system [2]. For the best systems, verification performance drops from $95 \%$ to $54 \%$ going indoors to outdoors.

To solve this difficult problem caused by variant lighting conditions, many approaches have been proposed. They can be divided into three categories. The first category includes those methods based on Lambertian model, which is too simply to describe the real face surface under various illuminations. Illumination Cone methods [3], quotient image based approaches $[4,5]$ belong to this category. Although these methods have achieved a degree of success in some face database such as Yale and CMU PIE, they need a large volume of training data under various illumination conditions. Another technique is using 3D data obtained from a laser scanner and 3D vision method [6]. Because the shape of 3D face is not affected by environmental lighting, such systems can obtain reasonable performance. The disadvantages are the increased cost, slowed speed and fussy 
pretreatment. The last category is using different imaging sensors in invisible spectral bands which can reduce the affection of ambient light. Thermal or far infrared imagery systems have advantages for detecting disguise faces, but they are subject to environment temperature, emotions and health conditions. In particular, infrared (IR) imaging sensor has become an area of growing interest [7]. Near-infrared (NIR) image based techniques have been developed to achieve highly accurate face recognition for indoor applications under varying lighting conditions $[8,9]$.

In this paper, we present a system for face recognition outdoor even in sunlight. Firstly, an enhanced near-infrared (ENIR) face imaging device is presented for outdoor face recognition. Then, algorithms are described for face detection and recognition in ENIR images. After that, an ENIR face recognition system is built. Experimental results show that the system performs slightly better than the existing NIR system $[8,9]$ while both are significantly better than visible light image based systems. In outdoor sunlight, the ENIR system can achieve relatively high recognition rate while NIR and visible light (VIS) systems cannot function properly.

The rest of the paper is organized as follows: Section 2 describes the design of the imaging hardware and presents an analysis of characteristics and amicability of resulting images for subsequent face processing. Section 3 discuses the face/eye detection and face recognition algorithms. Section 4 evaluates the system, including acquisition of testing database and comparison between VIS, NIR, ENIR systems. We will conclude in section 5 .

\section{Enhanced NIR Imaging}

The existing NIR based imaging hardware described in $[8,9]$ can produce face images of good quality for face recognition under indoor lighting. But outdoors, the environmental lighting contains much stronger NIR component, especially under sunlight. Under such circumstances, the existing NIR imaging cannot produce face images of proper quality.

The goal of making an ENIR hardware is to get rid of influence of strong NIR component under the outdoor sunlight. Like the NIR imaging device in $[8,9]$, we still use active light source mounted on the camera to provide frontal lighting and use filter to reduce environmental lighting. But to improve outdoor performance of the face recognition system, we make some revision on the basis of the NIR solution. The active light source must meet the following conditions: (1) In order to produce images of good quality, the strength of active light source must be stronger $1.5-2.5$ times than the strength of the sunlight. (2) The strength of active light source can not be too strong to disturb human eyes. (3) The resulting face images should be affected as little as possible when minimizing the sunlight.

Our solution uses the following strategies [10]:

1. Use a narrow band NIR laser generator, instead of LED, to provide active lighting;

2. Use very short exposure time (50ns), synchronized with the laser light; 
3. Use a narrow band pass optical filter on the camera lens to cut off visible light and sunlight while allowing NIR light to pass. The center frequency of optical filter is $808 \mathrm{~nm}$ as same as the laser generator and bandwith of the optical filter is $16 \mathrm{~nm}$.

4. Apply a differentiation between two successive frames. The first frame is captured by illuminating the subject's face with an active light source and the second is captured by turning this light source off. The differentiation operation can successfully eliminate NIR component in the static ambient light and sunlight [11].

As a result, this ENIR imaging hardware not only provides appropriate active frontal lighting but also minimizes ambient light as well as outdoor sunlight.

Fig. 1 shows the ENIR imaging device and some images captured when the active light source on/off and the finally output of the ENIR camera under the sunlight. From the third image in Fig. 1, we can see that ENIR imaging hardware can work properly under the sunlight. While active NIR illumination has been widely used in surveillance, face detection and face recognition $[12,13$, 11], the novelty of our hardware is to combine a strong active NIR light and differentiation technique together.

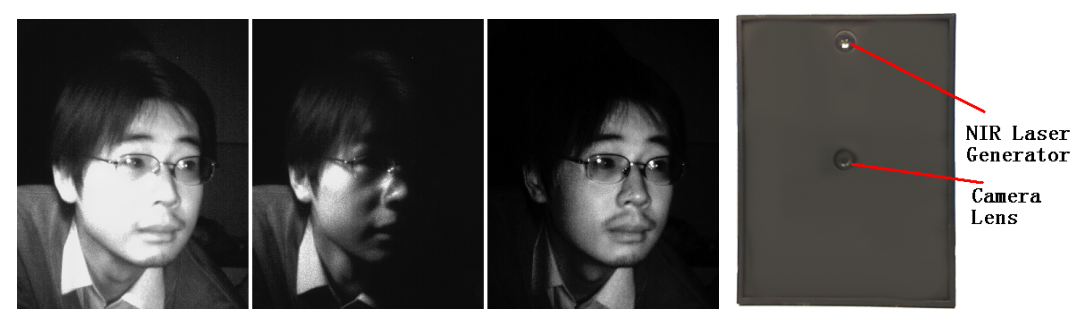

Fig. 1. From left to right: (a) Face image under mixture of sunlight and NIR light, (b) face image under sunlight only, (c) face image difference (a)-(b), (d) the ENIR imaging device

\section{Face/Eye Detection and Face Recognition}

In this system, face and eye detection is performed using an AdaBoost learning algorithm with an extended Haar feature [14]. A cascade form classifier is learned from face and non-face training data. For face detection, an example is a $20 \times 20$ image, containing a face or non-face pattern. Face detection in NIR or ENIR images is relatively easier than in VIS images, because the background of NIR or ENIR images is nearly black. On the contrary, detection of eyes in NIR or ENIR images is more challenging than in normal VIS images due to likely specular reflections on eyeglasses, eyelid occlusion, which happens among people in some 
ethnic groups and senior people and eye closing due to blinking are among other problems. Eye detection in these situations cannot be done by using a simple eye detector. To tackle the difficulties, a coarse to fine, easy to complex architecture is used to overcome the problem and achieve very high performance, see [9] for details.

For face recognition, we use statistical learning method based on AdaBoost [9] to build the classifier. Given a training set of local binary pattern (LBP) features [15] of faces subject to image noises, slight pose changes, and alignment errors, such a learning method performs a transform to the find most discriminative features and thereby builds a strong classifier. Because AdaBoost is usually used to solve two-class problem, we convert the multi-class problem to many two-class problems using intra-person and extra-person divergence [16]. Using AdaBoost learning, we select a number of most discriminative LBP histogram features to combine to a strong classifier. The procedure learns a sequence of $k$ weak classifiers, and linearly combines them into a stronger classifier as follow :

$$
H(x)=\operatorname{sign}\left(\sum_{t=1}^{T} \alpha_{t} h_{t}(x)\right) .
$$

In the test phase, the selected LBP features of two face images are matched, one for template face image and one for test face image. Some distance are taken between these two set of face images. And then each distance is input to weak classifier $h_{t}(x)$. At last, weak decisions are linearly combined with the weights $\alpha_{t}$ to give a final decision.

For the equality, VIS, NIR and ENIR systems all use the algorithms above for face/eye detection and face recognition. For VIS system, 2346 LBP histogram features are selected. For NIR and ENIR system, 1024 LBP histogram features are selected.

\section{System Evaluation}

\subsection{Database}

The purpose is to evaluate the ENIR face recognition system and compare it to a VIS and an NIR systems $[8,9]$. The classifier for each system is trained by 12000 face images containing 200 persons. A independent database of 20 persons was built for testing. The subjects were asked to face to our camera, about 50$100 \mathrm{~cm}$ to the device, presenting neutral and smile expressions. 10 face images were taken for each person, using 3 types of imaging devices (VIS, NIR and ENIR), for each of the following different lighting conditions:

1. normal indoor frontal lighting,

2. normal indoor non-frontal lighting,

3. strong indoor frontal lighting,

4. strong indoor non-frontal lighting,

5. outdoor frontal sunlight, 
6. outdoor side sunlight, and

7. outdoor back sunlight.

The strong indoor frontal lighting was produced using a tungsten-halogen lamp made by Mingzhu Livastore Industrial Co.,Ltd. The lamp has a color temperature of $2500-3000 \mathrm{~K}$, and spectral property as in Fig.2. It was $1000 \mathrm{~W}$ in power, placed at 3.5-4 meters away from the subject. The lamp contained a range of NIR composition and was powerful, thus well simulated the sunlight for indoor testing.

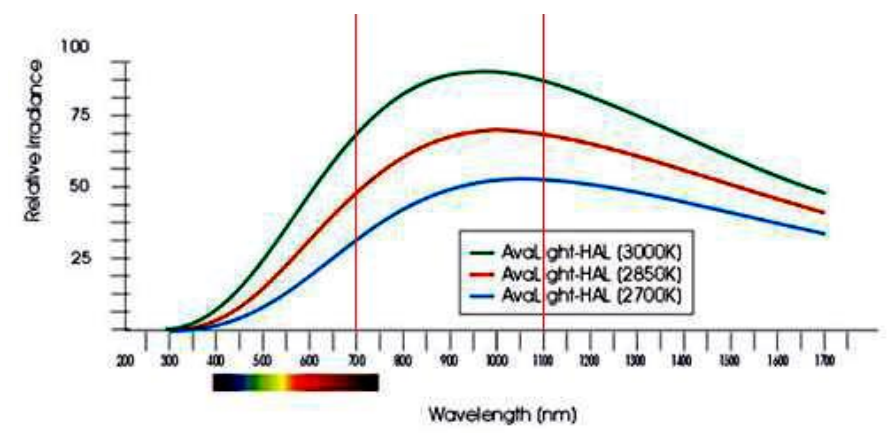

Fig. 2. Spectral property of the tungsten-halogen lamp.

The VIS and NIR cameras are saturated easily by sunlight, and so the database does not include VIS and NIR images outdoor. The number of VIS face images in this database is 4 (indoor conditions) $\times 20$ (people) $\times 10$ (images) $=800$ and we have the same amount of NIR face images. The amount of ENIR faces is $800+3$ (outdoor conditions) $\times 20$ (people) $\times 10$ (images) $=1400$. The resolution of VIS, NIR and ENIR cameras are all $640 \times 480$.

Fig. 3 shows some face image examples from the database. We can see that VIS face images were unstable to the direction and strength of lighting, NIR images were less unstable, and ENIR images were most stable to changes under various lighting condition.

\subsection{Performance}

The 3 systems (VIS, NIR and ENIR) were run in fully automatically for face detection, alignment and matching. Table 1 shows the face and eye detection rate of VIS, NIR and ENIR systems under different lighting conditions, where the " $\times$ " signs denote no performance measures due to unacceptable drop in performance. From the table, we see that the eye detection rate in VIS images dropped under halogen lamp light but not in a dreadful way; however, the eye detection rate in NIR images dropped acutely under the strong indoor nonfrontal lighting because the detector was trained using frontal NIR light only. 


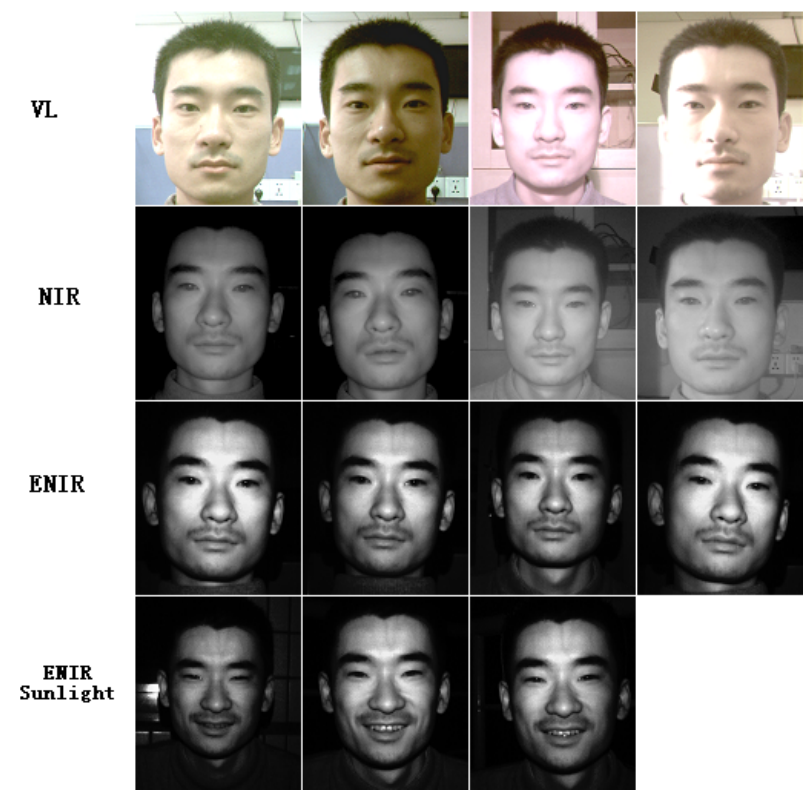

Fig. 3. Example face images in test database. Row 1-3: VIS, NIR and ENIR images under 4 indoor lighting conditions. Row 4: ENIR images outdoor.

As expected, the ENIR system has consistently good performance except under outdoor frontal sunlight; we are investigating the reasons.

Table 1. Face and eye detection rates under 7 lighting conditions

\begin{tabular}{|l|c|c|c|c|c|c|}
\hline \multirow{2}{*}{} & \multicolumn{2}{|c|}{ VIS } & \multicolumn{2}{c|}{ NIR } & \multicolumn{2}{c|}{ ENIR } \\
\cline { 2 - 7 } & Face & Eye & Face & Eye & Face & Eye \\
\hline Normal indoor frontal & $100 \%$ & $94.3 \%$ & $100 \%$ & $99.8 \%$ & $100 \%$ & $99.4 \%$ \\
\hline Normal indoor non-frontal & $100 \%$ & $92 \%$ & $100 \%$ & $99.5 \%$ & $100 \%$ & $97.2 \%$ \\
\hline Strong indoor frontal & $100 \%$ & $87.4 \%$ & $100 \%$ & $85.5 \%$ & $100 \%$ & $93.6 \%$ \\
\hline Strong indoor non-frontal & $100 \%$ & $91.6 \%$ & $91.6 \%$ & $38.4 \%$ & $100 \%$ & $98.5 \%$ \\
\hline Outdoor frontal sunlight & $\times$ & $\times$ & $\times$ & $\times$ & $100 \%$ & $75.5 \%$ \\
\hline Outdoor side sunlight & $\times$ & $\times$ & $\times$ & $\times$ & $100 \%$ & $96.6 \%$ \\
\hline Outdoor back sunlight & $\times$ & $\times$ & $\times$ & $\times$ & $100 \%$ & $95.5 \%$ \\
\hline
\end{tabular}

The database was divided into 2 sets, the gallery set and the probe set. The face images under normal indoor frontal lighting were used as the gallery images and the other images were used as probe images. Two protocols were used to compare the three systems: 
1. For indoor images taken under 4 lighting conditions: comparing recognition rates of all the 3 systems.

2. For outdoor images taken under 3 lighting conditions: evaluating recognition rates of the ENIR system only, while the other 2 systems could not function to a satisfactory extent.

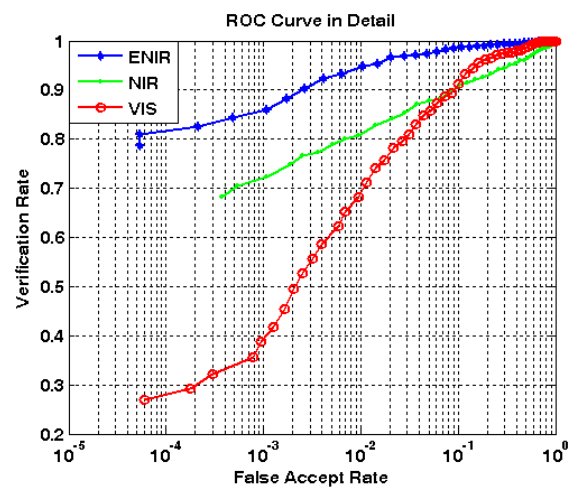

(a) Indoor frontal vs. Indoor non-frontal.

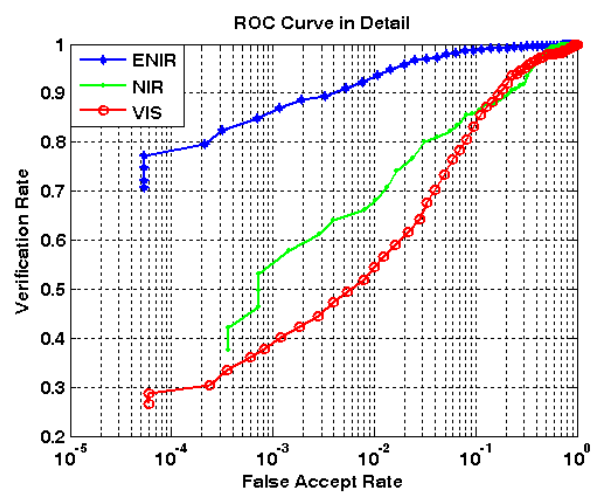

(c) Indoor frontal vs. Indoor strong non-frontal.

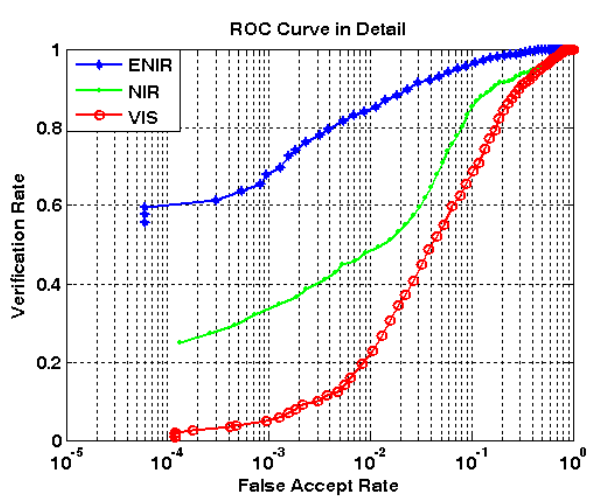

(b) Indoor frontal vs. Indoor strong frontal.

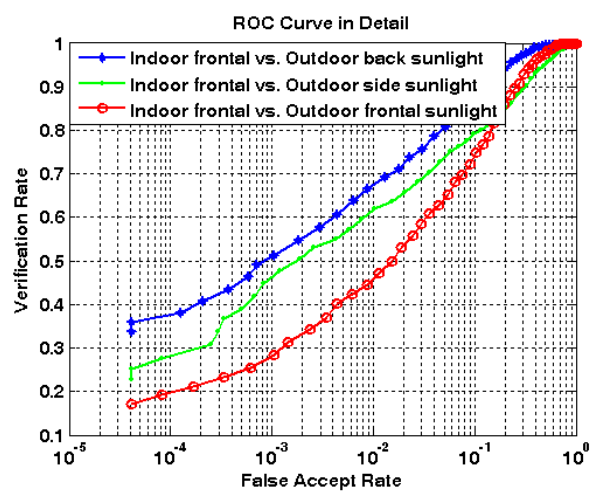

(d) ENIR system under outdoor sunlight.

Fig. 4. ROC curves for the test data sets.

Fig. 4 shows the ROC curves for the tests. The performance of the ENIR system was significantly higher than the other systems for all conditions. In Fig. 4(b), the performance of VIS and NIR systems dropped significantly under strong frontal halogen lamp light, whereas the ENIR system still has relatively high verification rate $(\mathrm{VR}=69 \%$ when $\mathrm{FAR}=0.001$ and $\mathrm{VR}=85 \%$ when FAR $=0.01$ ). Similarly in Fig. 4(c), the impact of strong frontal lighting was greater than that of strong non-frontal lighting. Fig. 4(d) shows the results of the ENIR system under outdoor sunlight in three different directions. The verification ac- 
curacy was the highest $(\mathrm{VR}=50 \%$ when $\mathrm{FAR}=0.001$ and $\mathrm{VR}=69 \%$ when FAR $=0.01)$ when the sunlight was from the back direction, and was the worst for frontal sunlight.

As a rough comparison: for the best face recognition systems in FRVT 2002, the recognition rate for faces captured outdoors, at a FAR of 0.01, was 50\% [2]. Hence, we conclude that overall, the ENIR system performed significantly better the VIS and NIR systems.

\section{Conclusion}

We have presented a system for both indoor and outdoor face recognition using a newly developed ENIR imaging device. Preliminary results show that the ENIR system performed significantly better than the VIS and NIR systems indoor and outdoor, especially under sunlight.

However, the system is not perfect when the lighting is very strong. This may be due to the relatively narrow dynamics of the cost-saving CMOS sensor used in the device, and also that the noise produced by the differentiation has certain influence on the results. In the future, we will take some measures to improve the quality of the images acquired by ENIR hardware and re-train face/eye detectors and classifier to optimize the system. Moreover, the system needs to be further tested on larger database.

\section{Acknowledgments}

This work was supported by the following funding resources: National Natural Science Foundation Project \#60518002, National Science and Technology Supporting Platform Project \#2006BAK08B06, National 863 Program Projects \#2006AA01Z192 and \#2006AA01Z193, Chinese Academy of Sciences 100 people project, and the AuthenMetric Collaboration Foundation.

\section{References}

1. Zhao, W., Chellappa, R., Phillips, P., Rosenfeld, A.: Face recognition: A literature survey. ACM Computing Surveys (2003) 399-458

2. NIST: Face Recognition Vendor Tests (FRVT). (http://www.frvt.org)

3. Georghiades, A.S., Belhumeur, P.N., Kriegman, D.J.: "From few to many: Illumination cone models for face recognition under variable lighting and pose". IEEE Transactions on Pattern Analysis and Machine Intelligence 23(6) (2001) 643-660

4. Shashua, A., Raviv, T.R.: "The quotient image: Class based re-rendering and recognition with varying illuminations". IEEE Transactions on Pattern Analysis and Machine Intelligence 23(2) (2001) 129-139

5. Wang, H.T., Li, S.Z., Wang, Y.S.: "Generalized quotient image". In: Proceedings of IEEE Computer Society Conference on Computer Vision and Pattern Recognition. (2004) 498-505 
6. Bowyer, K.W., Chang, Flynn, P.J.: "A survey of 3D and multi-modal 3d+2d face recognition". In: Proceedings of International Conference Pattern Recognition. (2004) 358-361

7. Kong, S.G., Heo, J., Abidi, B., Paik, J., Abidi, M.: "Recent advances in visual and infrared face recognition - A review". Computer Vision and Image Understanding 97(1) (2005) 103-135

8. Li, S.Z., and His Face Team: "AuthenMetric F1: A Highly Accurate and Fast Face Recognition System". ICCV2005 - Demos (2005)

9. Li, S.Z., Chu, R., Liao, S., Zhang., L.: "Illumination invariant face recognition using near-infrared images". IEEE Transactions on Pattern Analysis and Machine Intelligence 26(Special issu e on Biometrics: Progress and Directions) (2007)

10. AuthenMetric and CASIA: "An Image Acquisition Aparatus, and Method and System for Its applications in Face Recognition". Patent Application No.200710062725.3 (2007)

11. Hizem, W., Krichen, E., Ni, Y., Dorizzi, B., Garcia-Salicetti, S.: "Specific sensors for face recognition". In: Proceedings of IAPR International Conference on Biometric. Volume 3832. (2006) 47-54

12. Dowdall, J., Pavlidis, I., Bebis, G.: "Face detection in the near-IR spectrum". Image and Vision Computing 21 (2003) 565-578

13. Zou, X., Kittler, J., Messer, K.: "Ambient illumination variation removal by active near-ir imaging". In: Proceedings of IAPR International Conference on Biometric. (2006) 19-25

14. Li, S.Z., Zhang, Z.Q.: "FloatBoost learning and statistical face detection". IEEE Transactions on Pattern Analysis and Machine Intelligence 26(9) (2004) 1112-1123

15. Ahonen, T., Hadid, A., M.Pietikainen: "Face recognition with local binary patterns". In: Proceedings of the European Conference on Computer Vision, Prague, Czech (2004) 469-481

16. Moghaddam, B., Nastar, C., Pentland, A.: "A Bayesian similarity measure for direct image matching". Media Lab Tech Report No.393, MIT (1996) 